\title{
Prognostic effects of glycometabolism changes in lung adenocarcinoma: a prospective observational study
}

\author{
Yiwei Huang ${ }^{1 \#}$, Xiaodong Yang ${ }^{1 \#}$, Fenghao Sun ${ }^{1 \#}$, Tao Lu ${ }^{1}$, Guoshu Bi ${ }^{1}$, Jiaqi Liang ${ }^{1}$, Qihai Sui ${ }^{1,2}$, \\ Cheng Zhan ${ }^{1}$, Yu Shi ${ }^{1}$, Qun Wang ${ }^{1}$ \\ ${ }^{1}$ Department of Thoracic Surgery, Zhongshan Hospital, ${ }^{2}$ Eight-year Program Clinical Medicine, Shanghai Medical College, Fudan University, \\ Shanghai 200032, China \\ Contributions: (I) Conception and design: C Zhan, Y Shi; (II) Administrative support: Y Shi, Q Wang; (III) Provision of study materials or patients: Y \\ Huang, X Yang; (IV) Collection and assembly of data: T Lu, G Bi, J Liang, Q Sui; (V) Data analysis and interpretation: Y Huang, X Yang, F Sun; (VI) \\ Manuscript writing: All authors; (VII) Final approval of manuscript: All authors. \\ \#These authors contributed equally to this work. \\ Correspondence to: Cheng Zhan, PhD; Yu Shi, MD. Department of Thoracic Surgery, Zhongshan Hospital, Fudan University, No. 180, Fenglin Road, \\ Shanghai 200032, China. Email: czhan10@fudan.edu.cn; shi.yu@zs-hospital.sh.cn.
}

\begin{abstract}
Background: Changes in glycometabolism of cancer cells provides cancer cells with growth advantages, which are also of great value in the prognosis prediction of the patients with lung adenocarcinoma. However, currently available studies are controversial.

Methods: We successively collected 100 paired surgical specimens from patients with lung adenocarcinoma. The content of glycometabolic intermediates in tissues was tested by liquid chromatography-mass spectrometry. Follow-up was conducted every 6 months for patients enrolled in this study.

Results: There were significant differences in the contents of six intermediates, including glucose $(\mathrm{P}<0.0001)$, pyruvate $(\mathrm{P}=0.0009)$, lactate $(\mathrm{P}<0.0001)$, citrate $(\mathrm{P}=0.0001), \alpha$-ketoglutarate $(\mathrm{P}=0.0002)$, and fumarate $(\mathrm{P}=0.0096)$. For different TNM stages, the pyruvate content $(\mathrm{P}<0.001)$ and lactate content $(\mathrm{P}<0.001)$ in the II/III/IV stage cancer tissues were significantly higher than those in the stage I cancer tissues. The overall survival (OS) of patients with high levels of glucose $(\mathrm{P}=0.0034)$, pyruvate $(\mathrm{P}<0.0001)$, lactate $(\mathrm{P}=0.049)$, and citrate $(\mathrm{P}=0.024)$ in cancer tissues was significantly worse than that of patients with low levels. $\mathrm{N}$ stage $(\mathrm{P}<0.001)$ and the contents of pyruvate $(\mathrm{P}=0.033)$ were independent prognostic factors for the OS.

Conclusions: The contents of glucose, pyruvate, lactate, and citrate in cancer tissues are higher than that in para-carcinoma tissues, and the long-term survival decrease in patients with higher glucose, pyruvate, lactate, and citrate.
\end{abstract}

Keywords: Lung adenocarcinoma; glycometabolism changes; clinicopathological characteristics; prognosis; Warburg effect

Submitted Jul 25, 2019. Accepted for publication Oct 12, 2019.

doi: $10.21037 /$ tlcr.2019.10.18

View this article at: http://dx.doi.org/10.21037/tlcr.2019.10.18

\section{Introduction}

Following the publication of Warburg's pioneering research in the 1920's, numerous studies have been reported in the field of cancer glycolysis and lactate metabolism (1). The studies proposed that cancer can be considered as a metabolic disease in which cancer cells strive to complete their proliferation agenda in a microenvironment characterized by heterogeneous and fluctuating resource bioavailability. Although ATP produced by glycolysis is significantly less than that produced by oxidative phosphorylation, the intermediate products of glycolysis provide the carbon sources needed for rapid cell proliferation (2). The nutrients required for proliferating cells include not only ATP, but also nucleic acids, proteins, 
membrane phospholipids, and fatty acids. Glycolysis can provide small molecule precursors or intermediates that contribute to cell proliferation, such as intermediates for non-essential amino acids, acetyl-CoA, and nucleotidesynthesized ribose to meet the need for rapid DNA replication (3). Changes in the metabolic characteristics of cancer cells provide cancer cell growth advantages, which have an impact on prognoses. Understanding the effect of glycometabolism changes may also provide accurate patient stratifications and appropriate drug administration by clinicians. Currently, studies on the effect of glycometabolism changes in cancer cells on the prognosis are controversial (4). Therefore, a prospective study is needed to better reveal the effect of changes in glycometabolism of cancer cells on prognosis.

Cancer metabolism studies, especially glucose metabolism, have become an integral part of cancer biology, similar to signal transduction and transcription studies. Cancer cells show unrestricted growth, promoting their survival by metabolic adaptation. Favored glycolysis under aerobic conditions, or the Warburg effect, the transition from oxidative phosphorylation to glycolysis, accompanied by the accumulation of lactate by-products in the surrounding microenvironment, represents the most typical change observed in the metabolism of cancer cells (5). Studies of hypoxia, including the relationship between hypoxia and cancer etiology and prognosis, as well as the diagnosis and treatment of cancer, have been a subject of tumor metabolism and microenvironmental research for many years. Clinically, ${ }^{18} \mathrm{~F}$-deoxyglucose $\left({ }^{18} \mathrm{~F}-\mathrm{FDG}\right)$ is widely used to display tumors and their metastases because tumor tissues have a higher rate of glucose uptake than most normal tissues (6). Warburg's findings (1) are still significant in common cancer diagnostic tests employing ${ }^{18} \mathrm{~F}-\mathrm{FDG}$ positron emission tomography, which has high diagnostic accuracy.

Targeting tumor cell metabolism has become a successful approach to the prevention or treatment of cancer (7). Most efforts have focused on the use of small molecules to inhibit the function of metabolic enzymes, which has been comprehensively reviewed elsewhere (8). A key to selective killing of cancer cells lies in their dependence on glycolysis. Increasing evidence supports many glycolytic enzymes and transporters as candidates for cancer treatment. This study revealed the relationship between the changes of glycometabolism in cancer cells and the clinicopathological characteristics of patients, as well as the impact of metabolic intermediates on the prognosis of cancer patients, to better select therapeutic targets.

\section{Methods}

\section{Subjects}

We collected pathological specimens from patients with lung adenocarcinoma after operation. After homogenizing, the content of intermediate products of glycometabolism was determined by liquid chromatography tandem mass spectrometry (LC-MS/MS). All cases were diagnosed as lung adenocarcinoma by frozen pathology. By analyzing the relationship between the content of these metabolites and the prognosis of patients, the effect of these metabolites on the prognosis of patients was clarified. Patients with lung adenocarcinoma confirmed using frozen pathology specimens were included in a follow-up cohort. Survival analyses were performed to compare the relationship of glycometabolism product levels with OS (defined as period from date disease diagnosed till last follow-up date or death). This study was approved by The Institutional Review Committee of Zhongshan Hospital, Fudan University, Shanghai, China (Approval Number: B2014$017 \mathrm{R}$ ), and it has been registered in ClinicalTrials.gov (https://clinicaltrials.gov/; ID: NCT03641105). All patients enrolled in the study signed informed consent forms.

\section{Sample collection}

We first selected potential patients with lung adenocarcinoma based on imaging diagnosis, and collected surgical specimens postoperatively. Surgical specimens were taken from the cancer tissues and the adjacent tissues. After the lung adenocarcinoma was confirmed by frozen pathology results, the required surgical specimens were processed. Exclusion criteria were patients with other serious diseases or pathologically diagnosed as not lung adenocarcinoma. We collected 100 paired surgical specimens from patients with lung adenocarcinoma at the Department of Thoracic Surgery of Zhongshan Hospital, Fudan University (Shanghai, China), between January 2016 and April 2016.

\section{Sample preparation and testing}

We first weighed $0.08 \mathrm{~g}$ tissue from each sample and processed it into a tissue homogenate. Then, we added $200 \mu \mathrm{L}$ of methanol to tissue sample homogenate, vortexed 
the sample for $1 \mathrm{~min}$, placed the sample in a centrifuge at $4{ }^{\circ} \mathrm{C}$, and centrifuged at $16,200 \times \mathrm{g}$ for $4 \mathrm{~min}$. After centrifugation, the supernatant was analyzed by instrument (Instrument model: HPLC-MS/MS; Ultimate3000, API3200Q TRAP; Sciex, Framington, MA, USA). All of the standards used are listed in Table S1.

\section{Patient demographics}

After pathological diagnoses, clinicopathological characteristics and demographics of patients were recorded. The case report form (CRF) includes the eligibility checklist and case report form. CRF table was used to check whether the patients met the criteria for admission and to record the data of basic information, vital signs, medical history, laboratory examination, tumor assessment, pathological diagnosis and staging, and prognosis. For patients enrolled in this study cohort, we conducted a follow-up every 6 months.

\section{Statistical analysis}

Statistical analysis was performed using Prism software, version 7 (GraphPad, La Jolla, CA, USA) and R software, version 3.4.4 (R Foundation for Statistical Computing, Vienna, Austria) for Windows. The patients were divided into two groups according to the best cut-off values of glycometabolism product content detected in cancer tissues. Paired-sample $t$-tests were used to analyze the difference of paired data between the two groups. Independent sample $t$-tests and ANOVA analysis of variance were used to analyze the average difference between two groups of data and more than two groups of data, respectively. The survival curve was carried out by using the Kaplan-Meier method, and the log-rank test was used to compare the curve. A two-tailed $\mathrm{P}$ value $<0.05$ was considered to be a significant difference.

\section{Results}

Surgical specimens from 100 patients were randomly collected and their corresponding clinical information was recorded. From June 2016 to April 2019, we followed up the patients for three years. One hundred pairs of adjacent tissues and cancer tissues were analyzed by LC-MS/MS for the relative contents of intermediate products during glucose metabolism, including glucose, glucose-6-phosphate/fructose-6-phosphate/glucose-1- phosphate (G6P-F6P-G1P), fructose-1,6-diphosphate (F162P), glyceraldehyde 3-phosphate/dihydroxyacetone phosphate (G3P-DHAP), 3-phosphoglycerate (G-3P), 2-phosphoglycerate (2PGA), phosphoenolpyruvate, pyruvate, lactate, citrate, cis-aconitic acid, $\alpha$-ketoglutarate, succinate, fumarate, malate, and oxaloacetate. We found that the content of phosphoenolpyruvate was not too low to be detected, while the other products were effectively detected and are displayed in a histogram (Figure 1A). The histogram shows that there was a significant difference in the content of intermediate products of glucose metabolism. We further compared the contents of these glycometabolic intermediates in adjacent tissues and cancerous tissues with paired-sample $t$-tests, and found that there were significant differences in the contents of six intermediates (Figure 1B,C,D,E,F,G), namely, glucose $(\mathrm{P}<0.0001)$, pyruvate $(\mathrm{P}=0.0009)$, lactate $(\mathrm{P}<0.0001)$, citrate $(\mathrm{P}=0.0001), \alpha$-ketoglutarate $(\mathrm{P}=0.0002)$, and fumarate $(\mathrm{P}=0.0096)$. The contents of these six intermediate products increased significantly, except $\alpha$-ketoglutarate was decreased significantly in cancer tissues.

Univariate analyses were performed to identify the relationships between these intermediates and clinicopathological and demographic characteristics of the patients. As shown in Table 1, we found that there were significant differences in the contents of these glycometabolic intermediates according to sex and TNM stages, but not according to age, smoking history, or tumor location. The content of citrate in cancer tissues of male patients was significantly higher than that of female patients $(\mathrm{P}=0.014)$. For different TNM stages, the pyruvate content $(\mathrm{P}<0.001)$ and lactate content $(\mathrm{P}<0.001)$ in the II/III/IV stage cancer tissues were significantly higher than those in the stage I cancer tissues. The content of citrate $(\mathrm{P}=0.005)$ in the cancer tissues of stage II/III patients was significantly higher than that in the cancer tissues of stage I patients. However, the citrate content in the cancer tissues of stage IV patients was the lowest. In patients with different $T$ stages, lactate content $(\mathrm{P}=0.013)$ increased with increasing T stage. The levels of glucose $(\mathrm{P}=0.002)$, pyruvate $(\mathrm{P}<0.001)$, lactate $(\mathrm{P}<0.001)$, and citrate $(\mathrm{P}=0.003)$ in $\mathrm{N} 1 / \mathrm{N} 2$ stage patients were obviously higher than those in N0 stage patients. Patients with distant metastases had higher levels of lactate $(\mathrm{P}=0.029)$ in tumor tissues than those without distant metastases.

In order to analyze whether these different intermediates in paracancerous tissues $v s$. cancer tissues correlated with patient survival, we used receiver operating characteristic 

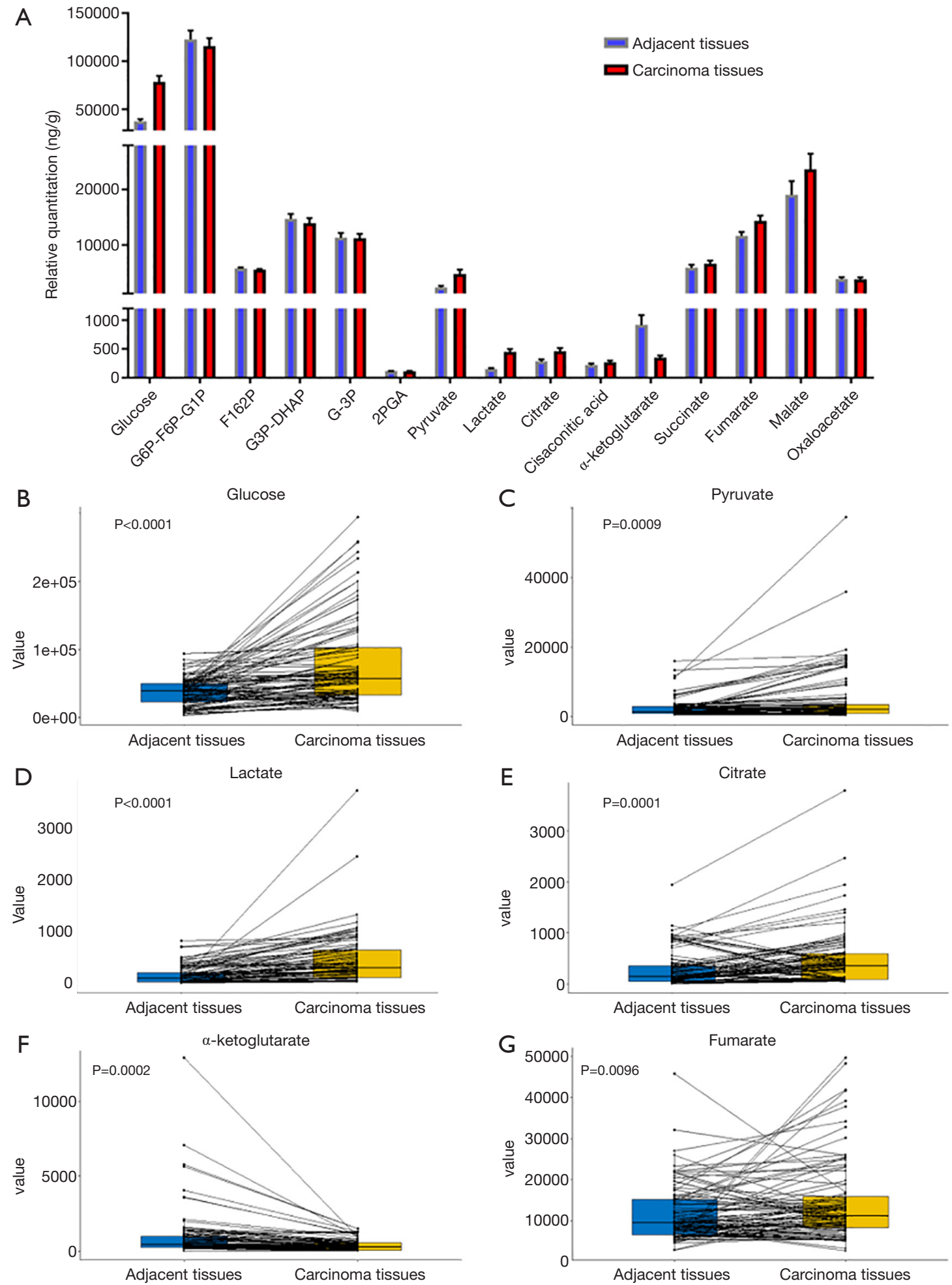

Figure 1 The changes of intermediate products of glucose metabolism in adjacent tissues and cancer tissues. (A) Relative contents of intermediate products during glucose metabolism in adjacent tissues and cancer tissues were analyzed by LC-MS/MS. The contents of (B) glucose, (C) pyruvate, (D) lactate, (E) citrate, (F) $\alpha$-ketoglutarate, and (G) fumarate in adjacent tissues and cancer tissues have significant difference. The $\mathrm{P}$ values were calculated using Paired-sample $t$-tests as shown. 
Table 1 Univariate analysis for the content difference of glucose metabolites in cancer tissue based on patients clinicopathologic characteristics

\begin{tabular}{|c|c|c|c|c|c|c|c|}
\hline \multirow{2}{*}{$\begin{array}{l}\text { Clinicopathologic } \\
\text { characteristics }\end{array}$} & \multirow{2}{*}{$\mathrm{N}$} & \multicolumn{6}{|c|}{ Standardized concentration of tumor samples (mean $\pm \mathrm{SD}, \mu \mathrm{g} / \mathrm{g}$ ) } \\
\hline & & Glucose & Pyruvate & Lactate & Citrate & Ketoglutarate & Fumarate \\
\hline Sex & 45 & & & & & & \\
\hline Men & 55 & $71,392.3 \pm 62,034.9$ & $7,207.8 \pm 13,212.0$ & $419.0 \pm 489.2$ & $848.8 \pm 1,273.1$ & $312.9 \pm 409.5$ & $14,720.8 \pm 10,781.2$ \\
\hline Women & & $76,966.7 \pm 58,494.8$ & $5,535.2 \pm 9,592.1$ & $497.5 \pm 545.5$ & $369.9 \pm 554.8$ & $379.9 \pm 362.0$ & $13,852.1 \pm 7,914.3$ \\
\hline $\mathrm{P}$ & & 0.646 & 0.466 & 0.455 & 0.014 & 0.388 & 0.644 \\
\hline Age & 63 & & & & & & \\
\hline$<65$ years & 37 & $66,436.9 \pm 49,613.4$ & $5,436.1 \pm 10,627.6$ & $397.1 \pm 347.7$ & $550.7 \pm 1,080.7$ & $361.6 \pm 380.6$ & $13,876.6 \pm 8,614.9$ \\
\hline$\geq 65$ years & & $88,116.1 \pm 72,895.7$ & $7,738.3 \pm 12,463.3$ & $572.9 \pm 717.5$ & $644.5 \pm 763.5$ & $329.5 \pm 393.1$ & $14,866.8 \pm 10,396.3$ \\
\hline $\mathrm{P}$ & & 0.080 & 0.329 & 0.103 & 0.644 & 0.689 & 0.609 \\
\hline Smoking history & 74 & & & & & & \\
\hline Never & 26 & $70,753.1 \pm 56,079.8$ & $5,310.0 \pm 9,421.4$ & $430.1 \pm 506.7$ & $557.4 \pm 1,025.6$ & $335.0 \pm 337.4$ & $14,820.8 \pm 9,480.2$ \\
\hline Former/current & & $85,003.4 \pm 69,678.1$ & $9,071.1 \pm 15,448.5$ & $553.5 \pm 555.4$ & $665.1 \pm 814.8$ & $391.6 \pm 497.8$ & $12,598.5 \pm 8,624.4$ \\
\hline $\mathrm{P}$ & & 0.299 & 0.146 & 0.300 & 0.629 & 0.520 & 0.296 \\
\hline Tumor location & 19 & & & & & & \\
\hline Left upper & 28 & $81,408.9 \pm 49,996.3$ & $6,633.9 \pm 9,250.6$ & $458.7 \pm 329.4$ & $639.2 \pm 685.1$ & $261.3 \pm 285.8$ & $12,782.6 \pm 5,948.9$ \\
\hline Left lower & 33 & $66,849.1 \pm 59,362.9$ & $7,808.7 \pm 15,755.1$ & $440.2 \pm 514.6$ & $332.8 \pm 347.7$ & $273.0 \pm 294.3$ & $14,322.8 \pm 9,406.4$ \\
\hline Right upper & 11 & $76,790.2 \pm 69,982.4$ & $5,539.9 \pm 10,739.2$ & $498.7 \pm 683.3$ & $693.5 \pm 1,428.7$ & $376.5 \pm 423.2$ & $14,607.1 \pm 10,693.9$ \\
\hline Right middle & 9 & $90,060.2 \pm 60,906.4$ & $6,917.4 \pm 7,024.4$ & $532.8 \pm 405.7$ & $591.2 \pm 552.9$ & $402.0 \pm 441.1$ & $11,411.9 \pm 7,102.2$ \\
\hline Right lower & & $55,837.8 \pm 39,354.5$ & $2,799.5 \pm 3,400.5$ & $317.6 \pm 308.4$ & $853.7 \pm 1153.8$ & $612.7 \pm 511.1$ & $19,202.9 \pm 11,015.6$ \\
\hline $\mathrm{P}$ & & 0.675 & 0.824 & 0.896 & 0.559 & 0.150 & 0.395 \\
\hline TNM stage & 73 & & & & & & \\
\hline 1 & 9 & $65,186.5 \pm 50,048.2$ & $3,375.3 \pm 4,564.2$ & $357.8 \pm 312.6$ & $414.9 \pm 554.8$ & $372.4 \pm 376.5$ & $14,178.0 \pm 9,062.3$ \\
\hline II & 15 & $99,192.6 \pm 85,629.9$ & $11,160.1 \pm 18,354.7$ & $979.6 \pm 1,082.8$ & $743.6 \pm 1,212.6$ & $387.1 \pm 483.9$ & $10,689.3 \pm 6,685.8$ \\
\hline III & 3 & $93,207.9 \pm 66,571.5$ & $15,742.2 \pm 19,703.1$ & $531.4 \pm 453.2$ & 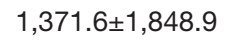 & $280.1 \pm 383.3$ & $18,138.5 \pm 11,157.7$ \\
\hline IV & & $132,119.6 \pm 115,721.1$ & $15,273.6 \pm 18,335.0$ & $1,103.1 \pm 1,214.3$ & $327.3 \pm 48.7$ & $32.6 \pm 47.9$ & $7,007.0 \pm 1238.6$ \\
\hline $\mathrm{P}$ & & 0.054 & $<0.001$ & $<0.001$ & 0.005 & 0.415 & 0.121 \\
\hline T stage & 63 & & & & & & \\
\hline $\mathrm{T} 1$ & 35 & $67,324.9 \pm 55,193.2$ & $5,679.5 \pm 10,950.4$ & $387.7 \pm 363.8$ & $503.8 \pm 640.6$ & $378.8 \pm 419.9$ & $14,499.8 \pm 10,333.6$ \\
\hline $\mathrm{T} 2$ & 2 & $82,514.0 \pm 60,993.5$ & $6,655.7 \pm 11,324.4$ & $543.6 \pm 641.1$ & $733.8 \pm 1,402.8$ & $311.6 \pm 315.5$ & $14,220.4 \pm 7,215.7$ \\
\hline T3 & & $158,182.3 \pm 141,459.4$ & $19,015.4 \pm 23,904.9$ & $1,383.9 \pm 1,512.2$ & $558.8 \pm 251.2$ & $100.7 \pm 142.4$ & $6,547.9 \pm 1,370.7$ \\
\hline $\mathrm{P}$ & & 0.065 & 0.256 & 0.013 & 0.537 & 0.465 & 0.495 \\
\hline $\mathrm{N}$ stage & 73 & & & & & & \\
\hline No & 11 & $62,090.6 \pm 44,741.7$ & $3,123.6 \pm 4,165.3$ & $340.9 \pm 291.9$ & $418.0 \pm 553.5$ & $355.9 \pm 361.8$ & $14,099.2 \pm 9,092.9$ \\
\hline N1 & 16 & $114,261.6 \pm 90,031.8$ & $11,701.2 \pm 16,619.6$ & $991.3 \pm 977.3$ & $642.6 \pm 1,109.1$ & $435.1 \pm 529.9$ & $10,673.1 \pm 6,123.0$ \\
\hline N2 & & $103,520.4 \pm 76,406.2$ & $17,003.2 \pm 19,692.0$ & $651.5 \pm 650.0$ & $1,309.7 \pm 1,803.3$ & $262.6 \pm 376.9$ & $17,353.5 \pm 11,227.4$ \\
\hline $\mathrm{P}$ & & 0.002 & $<0.001$ & $<0.001$ & 0.003 & 0.504 & 0.179 \\
\hline M stage & 97 & & & & & & \\
\hline MO & 3 & $72,674.9 \pm 57,536.1$ & $6,010.0 \pm 11,085.0$ & $442.3 \pm 484.4$ & $593.4 \pm 986.4$ & $359.5 \pm 385.3$ & $14,466.8 \pm 9,326.4$ \\
\hline M1 & & $132,119.6 \pm 115,721.1$ & $15,273.6 \pm 18,335.0$ & $1,103.1 \pm 1,214.3$ & $327.3 \pm 48.7$ & $32.6 \pm 47.9$ & $7,007.0 \pm 1,238.6$ \\
\hline $\mathrm{P}$ & & 0.090 & 0.164 & 0.029 & 0.643 & 0.147 & 0.171 \\
\hline
\end{tabular}

SD, standard deviation. 
A

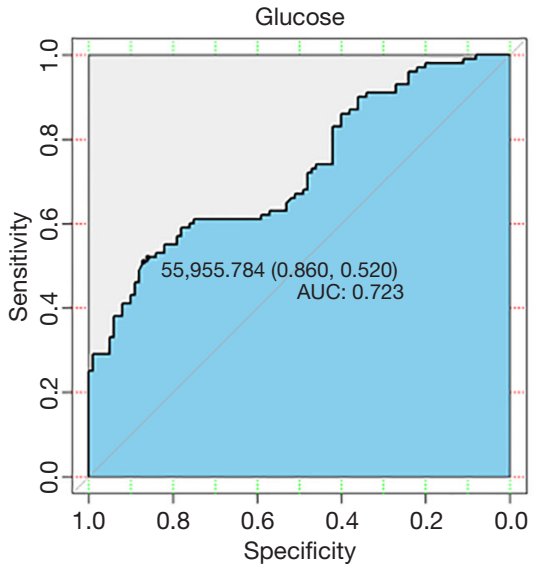

D

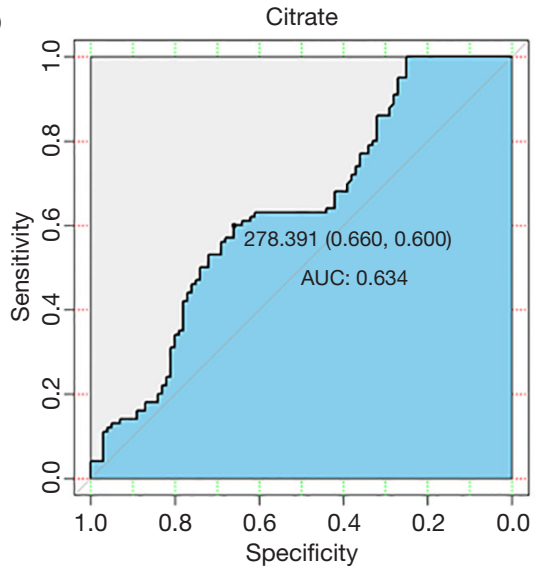

B

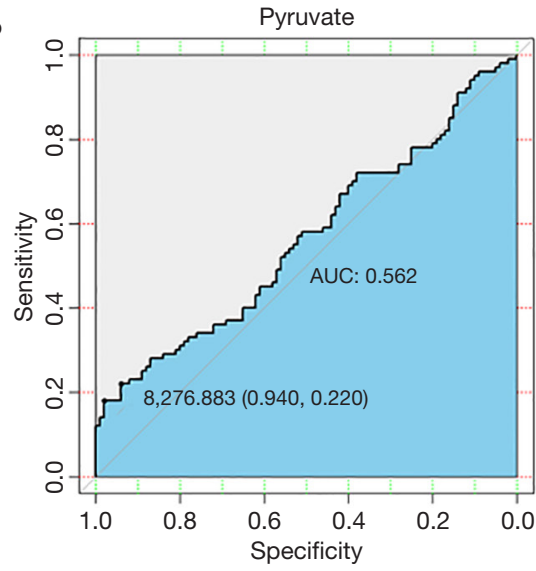

$\mathrm{E}$

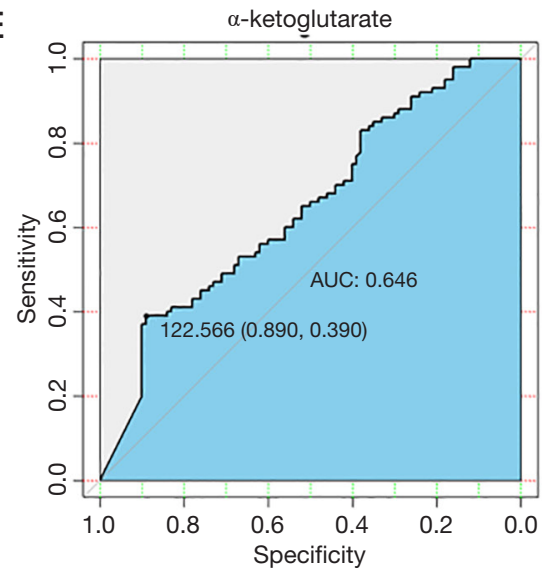

C

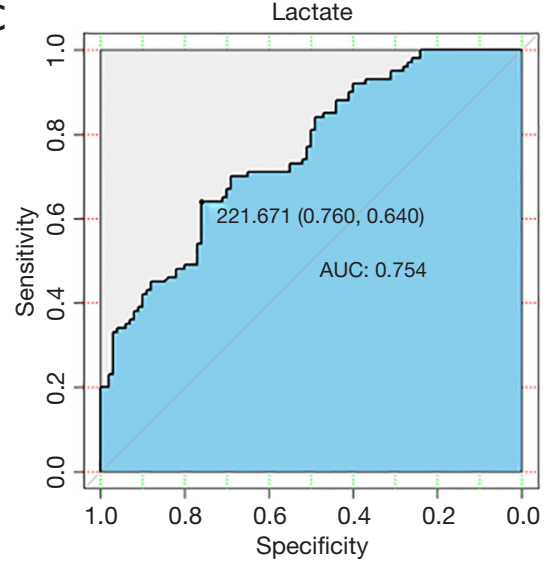

$\mathrm{F}$

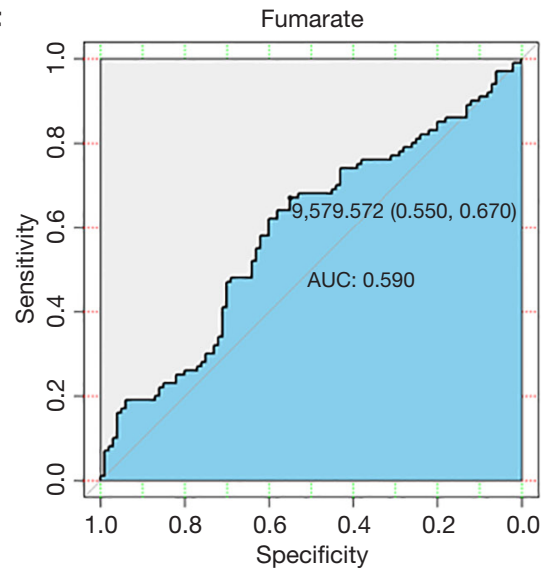

Figure 2 The best cut-off values and the area under the curve values of (A) glucose, (B) pyruvate, (C) lactate, (D) citrate, (E) $\alpha$-ketoglutarate, and (F) fumarate obtained using receiver operating characteristic curves.

curves to obtain the best cut-off values of these metabolites in tumor tissues. As shown in Figure 2, the optimal cut-off values of glucose, pyruvate, lactate, citrate, $\alpha$-ketoglutarate, and fumarate in tumor tissues were 55,955.784, 8,276.883, $221.671,278.391,122.566$, and $9,579.572 \mathrm{ng} / \mathrm{mg}$, respectively, and the area under the curve values were $0.723,0.562,0.754,0.634,0.646$, and 0.590 , respectively. Then, the correlations of the contents of these intermediate products with survival were analyzed. As shown in Figure 3, the survival of patients with high levels of glucose $(\mathrm{P}=0.0034)$, pyruvate $(\mathrm{P}<0.0001)$, lactate $(\mathrm{P}=0.049)$, and citrate $(\mathrm{P}=0.024)$ in cancer tissues was significantly worse than that of patients with low levels of these metabolites. The level of pyruvate had the greatest correlation with survival. However, the levels of $\alpha$-ketoglutarate and fumarate had no influence on patient survival.

Univariate analyses of OS showed that the patient's tumor $\mathrm{N}$ stage $(\mathrm{P}<0.001)$, $\mathrm{M}$ stage $(\mathrm{P}<0.001)$, and the content of glucose $(\mathrm{P}=0.012)$, pyruvate $(\mathrm{P}<0.001)$, as well as citrate $(\mathrm{P}=0.041)$ in tumor tissues were significant predictors of the outcome (Table S2). Furthermore, all of the variables whose $P$ value $<0.1$ in univariate analyses were included in the multivariate cox analyses for OS. Finally, we conferred that $\mathrm{N}$ stage $(\mathrm{P}<0.001)$ and pyruvate $(\mathrm{P}=0.033)$ were independent prognostic factors for the OS (Figure 4). A nomogram (Figure $5 \mathrm{~A}$ ) was then constructed according to all significant risk factors identified by the multivariate analyses for predicting 1 - and 3-year OS with the area under the curve values as 0.907 and 0.909 (Figure 5B,C). The $\mathrm{C}$-index for prediction was 0.871 . The calibration plot of this nomogram was also provided in Figure S1.

\section{Discussion}

Inhibition of aerobic glycolysis has attracted attention in the treatment of cancer. Many key proteins in the 

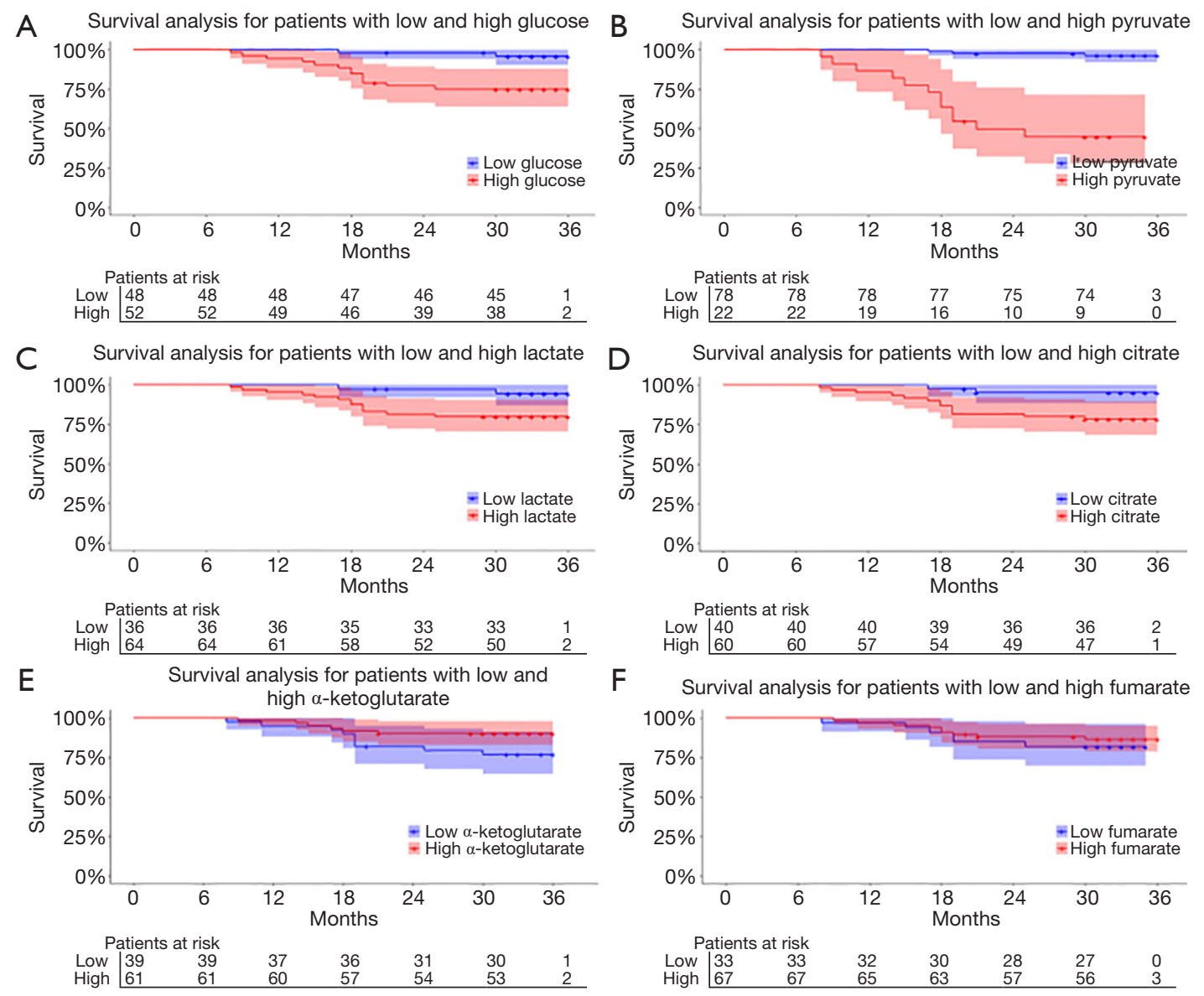

Figure 3 Survival analyses for patients with high and low contents of (A) glucose, (B) pyruvate, (C) lactate, (D) citrate, (E) $\alpha$-ketoglutarate, and $(\mathrm{F})$ fumarate in cancer tissues.

glycolytic pathway have been identified as important targets for treatment and overcoming drug resistance. Most of these proteins are enzymes in the carbohydrate metabolism pathway. Targeting these enzymes can affect glycometabolism and other substances synthesized by glycometabolism intermediates in cancer cells. Generally, tumor cells maintain a state of high glucose metabolism. Compared with normal cells, glucose deprivation can significantly increase levels of reactive oxygen species in cancer cells, resulting in energy stress and selective death of cancer cells (9). A study showed that high glucose increased cancer cell growth and improved the ability of adhesion and invasiveness. In addition, high glucose increased glucose uptake and glycolytic activity by regulating the AMPK/ mTOR/S6 and MAPK pathways (10). Consistent with previous studies, our results showed that the content of glucose in cancer tissues was significantly higher than that in paracancerous tissues. With the increase of glucose content in cancer tissue, the survival of patients was decreased.

Intermediate products of glycolysis can affect the survival of cancer cells, such as G6P, G3P, and pyruvate. Pyruvate is important for cancer metabolism. This molecule is produced by glucose catabolism as the final product of glycolysis or can be taken into cells. Previous studies on mitochondrial function, cell proliferation, and invasiveness showed that breast cancer cells were more invasive in the presence of exogenous pyruvate than in the presence of glucose and lactate as the main energy sources (11). Pyruvate can provide fuel for mitochondrial oxygen consumption and respiratory reserve capacity. The increase of mitochondrial metabolism is related to cell proliferation and invasiveness in vitro (11). A study has shown that pyruvate in the nutritional microenvironment can promote proliferation by supporting anaplerosis without increasing mitochondrial oxidative metabolism (12). In addition, researchers found that the level of serum pyruvate in 


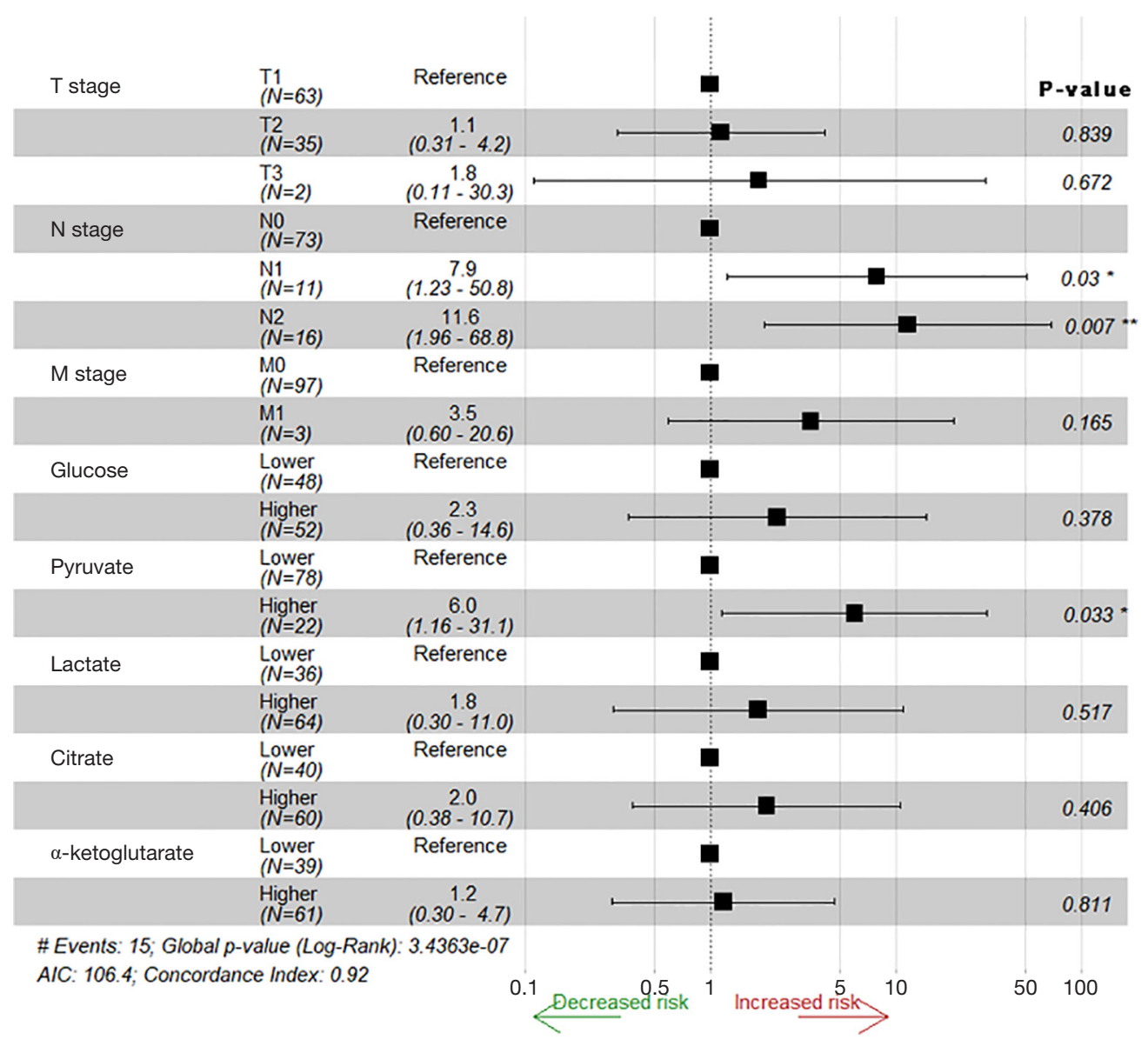

Figure 4 Forest plots based on multivariate cox analyses for overall survival. *, $\mathrm{P}<0.05 ;{ }^{* *}, \mathrm{P}<0.01$.

patients with stage IV lung cancer was higher than that in patients with stage I lung cancer (13), which was consistent with our results. Survival analyses also showed that the increase of pyruvate in cancer tissue significantly correlated with reduced overall survival of patients.

Lactate can be oxidized to pyruvate and input into the TCA cycle or used as a source of gluconeogenesis in the liver. The lactate produced by glycolysis reduces the $\mathrm{pH}$ of the extracellular matrix (14). An acidic microenvironment can promote the ability of tumor invasion and metastasis (15). Lactate itself is an endogenous inflammatory mediator that causes $\mathrm{T}$ cells and macrophages to produce interleukin$17 \mathrm{~A}$, thereby promoting chronic inflammation in the tumor microenvironment (16). When tumor cells release large amounts of lactate into the extracellular space, the immune cells cannot exclude their own lactate because the secretion of cellular lactate depends on the ratio of intracellular and extracellular concentrations. Recent studies support the transforming growth factor- $\beta 2$ signaling pathway as an important mediator of lactate-associated cancer cell migration (17). An independent study of patients with head and neck squamous cell carcinoma also showed a negative correlation between pretreatment lactate concentration in a single primary tumor and overall survival, as well as diseasefree survival (18). Similarly, magnetic resonance imaging studies of glioblastoma also showed a negative correlation between high lactate concentration, relative to normal brain tissue, and survival of patients (19). Increased lactate concentration is associated with lymph node metastasis or distant metastasis in patients with head and neck cancer. This more aggressive malignant phenotype may be related to hypoxia-mediated radioresistance and upregulation of metastasis-related genes (20). In this study, we also found that lactate levels in cancer tissues were elevated in patients with lymph node metastasis and distant metastasis. These findings confirmed the dual role of lactate in cancer: it acts as both a metabolic fuel and a signaling molecule, locating lactate at the intersection of the key processes of cancer 
$A$

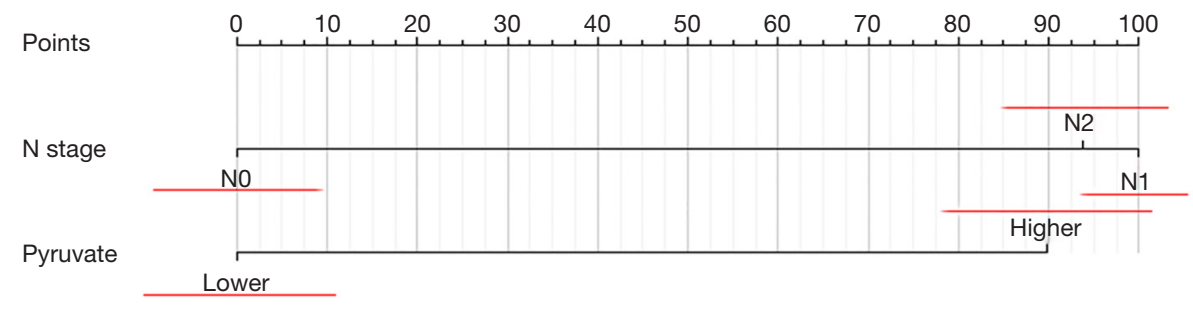

Total points

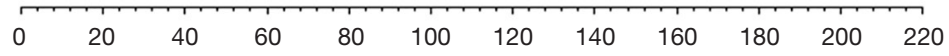

1-year survival

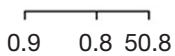

3-year survival
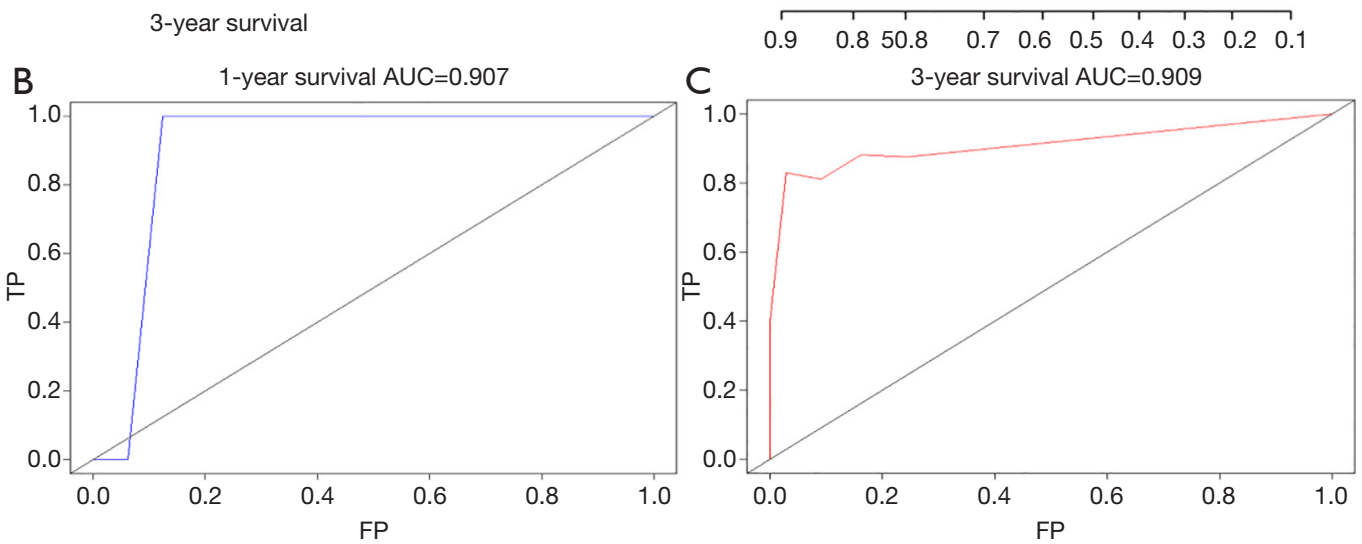

Figure 5 A nomogram for predicting 1- and 3-year overall survival for patients with lung adenocarcinoma (A) with the receiver operating characteristic curves (B,C).

progression, namely, tumor metabolism and angiogenesis. These effects of lactate make the prognosis of patients with higher lactate content worse, as revealed by our results that the higher the lactate content, the worse the prognosis.

Citrate is an intermediate in the TCA cycle, which accumulates in tissues when the rate of glycolysis exceeds the activity of the TCA cycle (21). Even in the presence of oxygen, proliferating cells reduce their oxidative metabolism and rely more on glycolysis (Warburg effect). This metabolic shift reduces citrate biosynthesis and intracellular acidity, both of which promote glycolysis to maintain tumor growth. Because citrate is a donor of acetyl CoA, its reduced production is conducive to the deacetylation of proteins favoring anti-apoptotic and epigenetic changes, both of which contribute to the invasiveness of tumors. Experimental studies also have shown that citrate can inhibit the growth of tumors, reverse cell dedifferentiation, and increase sensitivity to cisplatin (22). It was reported that dietary citrate supplementation may be beneficial for cancer treatment (23). Superfluous citrate can be used by cells to transfer mitochondrial acetyl-CoA to the cytoplasm for fatty acid biosynthesis, which is ultimately necessary for the ab initio synthesis of cell membranes. This is an important step in supporting cell division and increasing the biomass of growing tumors (24). There is evidence that citrate may play a role in tumor biology, and that the decrease in citrate concentration in tumor cells may be related to tumor aggressiveness (25). Citrate levels could be used as indicators of cancer invasiveness and/or as biomarkers of response to treatment. However, in this study, we found no difference in citrate levels between patients with and without metastasis. In cancer samples with the same number of benign epithelium and stroma, the studies found that citrate levels in high risk samples were lower than those in low risk samples (4). This was contrary to our results, which showed that citrate was higher in cancer tissues than adjacent tissues. At the same time, our results also showed that the higher the level of citrate, the worse the long-term survival of patients. These contradictory conclusions need to be further studied. 
As reported, adding alpha-ketoglutarate can increase the levels of tumor suppressor succinate dehydrogenase (SDH) and fumarate hydratase, decreasing the metabolites of succinate and fumarate, further stabilizing the hydroxylase 2 of hypoxia inducible factor-1 (HIF-1 $\alpha$ ) and decreasing HIF- $1 \alpha$, ultimately inhibiting the metastasis of breast cancer (26). Moreover, in vitro and in vivo experiments confirmed that the accumulation of alpha-ketoglutarate was correlated with the increase of nitric oxide. This epigenetic modification of the metabolic environment effectively counteracts the initial steps of tumor invasion and inhibits the transformation of epithelial cells into mesenchymal cells (27). In this study, we did not find a correlation between alpha-ketoglutarate and metastasis. In addition, although the contents of alpha-ketoglutarate and fumarate were different in cancer tissues vs. paracancerous tissues, the levels of alpha-ketoglutarate and fumarate in cancer tissues did not significantly affect the long-term survival of patients. However, previous studies have shown that the epigenetic changes of miRNA clustermir-200ba429, an antimetastasis microRNA cluster, can lead to the accumulation of fumarate in renal cancer with a deficiency of fumarate hydratase, thus conferring the tumor invasiveness and tumorigenesis (28), which was not found in our research.

Other intermediates of the TCA cycle are also thought to affect tumor growth and patient prognoses. Succinate was reported to not only promote cancer, but also has anti-cancer effects. The SDH mutation results in the accumulation of succinate and subsequent stabilization of HIF-1 $\alpha$, thus providing a growth advantage for tumors (29). The depletion of malic enzyme 2 (ME2) results in the accumulation of malate and the decrease of pyruvate. Effects of permeable dimethyl-malate (DMM) of exogenous cells is similar to the ME2 knockout phenotype, and both $M E 2$ gene knockout and DMM treatment can decrease the growth of A549 cells in vivo (30). All these data need to be further explored.

To our knowledge, this is the first time that a large number of surgical specimens have been obtained to determine the content of glucose metabolic intermediates in these tissues, and a prospective clinical study has been conducted based on these results. This study may be helpful to understand the changes of glucose metabolism in cancer cells and the impact of these changes on the prognosis of patients, thus contributing to future studies to better choose the target of cancer treatment.

There were some limitations in our study. First, the data of tissue glycometabolism intermediates measured in this study varied slightly. The sampling and processing procedures should be standardized as much as possible to solve this problem. Second, if a larger sample size can be obtained, it will help to provide more reliable conclusions. In addition, with the increase of health awareness, the detection rate of early lung cancer has increased, resulting in a high proportion of stage I lung cancer patients in this study, which may cause some deviations in the data analyses. The AUC values from our data analysis are not very good, perhaps due to the limit number of samples. Exactly, there are many other factors that can affect the prognosis of lung cancer. Because of the limitations of the data we can obtain, many other variables, such as genetic mutation, operation methods, as well as social and economic conditions, have not been included in this study, which needs further improvement in the follow-up study.

\section{Conclusions}

$\mathrm{N}$ stage and the contents of pyruvate were independent prognostic factors for the OS of patients with lung adenocarcinoma. The contents of glucose, pyruvate, lactate, citrate and fumarate in cancer tissues were significantly higher than those in paracancerous tissues, while $\alpha$-ketoglutarate levels decreased. The long-term survival of patients decreased with the increase of glucose, pyruvate, lactate, and citrate in cancer tissues.

\section{Acknowledgments}

We thank the International Science Editing Co. for editing the language.

Funding: This work was supported by the National Natural Science Foundation of China [Grant number 81672268) (www.nsfc.gov.cn/)] and Research and Development Fund of Zhongshan Hospital, Fudan University (Grant number 2018ZSFZ002 and 2016ZSFZ56).

\section{Footnote}

Conflicts of Interest: The authors have no conflicts of interest to declare.

Ethical Statement: The authors are accountable for all aspects of the work in ensuring that questions related to the accuracy or integrity of any part of the work are appropriately investigated and resolved. This study was approved by The Institutional Review Committee 
of Zhongshan Hospital, Fudan University, Shanghai, China (Approval Number: B2014-017R), and it has been registered in ClinicalTrials.gov (https://clinicaltrials.gov/; ID: NCT03641105). All patients enrolled in the study signed informed consent forms.

\section{References}

1. Warburg O. On the origin of cancer cells. Science 1956;123:309-14.

2. Brooks GA. Cell-cell and intracellular lactate shuttles. J Physiol 2009;587:5591-600.

3. Vander Heiden MG, Cantley LC, Thompson CB. Understanding the Warburg effect: the metabolic requirements of cell proliferation. Science 2009;324:1029-33.

4. Bertilsson $\mathrm{H}$, Tessem MB, Flatberg A, et al. Changes in gene transcription underlying the aberrant citrate and choline metabolism in human prostate cancer samples. Clin Cancer Res 2012;18:3261-9.

5. Cairns RA, Harris IS, Mak TW. Regulation of cancer cell metabolism. Nat Rev Cancer 2011;11:85-95

6. Jung KH, Lee KH. Cancer therapy by targeting the Warburg effect with miR-143 and response monitoring with 18F-FDG PET. Transl Cancer Res 2017;6:S519-21.

7. Vander Heiden MG. Targeting cancer metabolism: a therapeutic window opens. Nat Rev Drug Discov 2011;10:671-84.

8. Granchi C, Minutolo F. Anticancer agents that counteract tumor glycolysis. Chemmedchem 2012;7:1318-50.

9. Nogueira V, Hay N. Molecular Pathways: Reactive Oxygen Species Homeostasis in Cancer Cells and Implications for Cancer Therapy. Clin Cancer Res 2013;19:4309.

10. Han J, Zhang L, Guo H, et al. Glucose promotes cell proliferation, glucose uptake and invasion in endometrial cancer cells via AMPK/mTOR/S6 and MAPK signaling. Gynecol Oncol 2015;138:668-75.

11. Diers AR, Broniowska KA, Chang CF, et al. Pyruvate fuels mitochondrial respiration and proliferation of breast cancer cells: effect of monocarboxylate transporter inhibition. Biochem J 2012;444:561-71.

12. Christen S, Lorendeau D, Schmieder R, et al. Breast Cancer-Derived Lung Metastases Show Increased Pyruvate Carboxylase-Dependent Anaplerosis. Cell Rep 2016;17:837-48.

13. Hong CS, Graham NA, Gu W, et al. MCT1 Modulates Cancer Cell Pyruvate Export and Growth of Tumors that Co-express MCT1 and MCT4. Cell Rep
2016;14:1590-601.

14. Held-Warmkessel J, Dell DD. Lactic acidosis in patients with cancer. Clin J Oncol Nurs 2014;18:592-4.

15. Peppicelli S, Bianchini F, Calorini L. Extracellular acidity, a "reappreciated" trait of tumor environment driving malignancy: perspectives in diagnosis and therapy. Cancer Metastasis Rev 2014;33:823-32.

16. Yabu M, Shime H, Hara H, et al. IL-23-dependent and -independent enhancement pathways of IL-17A production by lactic acid. Int Immunol 2011;23:29-41.

17. Baumann F, Leukel P, Doerfelt A, et al. Lactate promotes glioma migration by TGF-beta2-dependent regulation of matrix metalloproteinase-2. Neuro Oncol 2009;11:368-80.

18. Ziebart T, Walenta S, Kunkel M, et al. Metabolic and proteomic differentials in head and neck squamous cell carcinomas and normal gingival tissue. J Cancer Res Clin Oncol 2011;137:193-9.

19. Park I, Larson PE, Zierhut ML, et al. Hyperpolarized 13C magnetic resonance metabolic imaging: application to brain tumors. Neuro Oncol 2010;12:133-44.

20. Brizel DM, Schroeder T, Scher RL, et al. Elevated tumor lactate concentrations predict for an increased risk of metastases in head-and-neck cancer. Int J Radiat Oncol Biol Phys 2001;51:349-53.

21. Blüml S, Panigrahy A, Laskov M, et al. Elevated citrate in pediatric astrocytomas with malignant progression. Neuro Oncol 2011;13:1107-17.

22. Icard P, Lincet $\mathrm{H}$. The reduced concentration of citrate in cancer cells: An indicator of cancer aggressiveness and a possible therapeutic target. Drug Resist Updat 2016;29:47-53.

23. Ren JG, Seth P, Ye H, et al. Citrate Suppresses Tumor Growth in Multiple Models through Inhibition of Glycolysis, the Tricarboxylic Acid Cycle and the IGF-1R Pathway. Sci Rep 2017;7:4537.

24. Vander Heiden MG, Cantley LC, Thompson CB. Understanding the Warburg effect: the metabolic requirements of cell proliferation. Science 2009;324:1029-33

25. Icard $\mathrm{P}$, Lincet $\mathrm{H}$. The reduced concentration of citrate in cancer cells: An indicator of cancer aggressiveness and a possible therapeutic target. Drug Resist Updat 2016;29:47-53.

26. Tseng CW, Kuo WH, Chan SH, et al. Transketolase Regulates the Metabolic Switch to Control Breast Cancer Cell Metastasis via the alpha-Ketoglutarate Signaling Pathway. Cancer Res 2018;78:2799-12.

27. Atlante S, Visintin A, Marini E, et al. alpha-ketoglutarate 
dehydrogenase inhibition counteracts breast cancerassociated lung metastasis. Cell Death Dis 2018;9:756.

28. Fuhler GM, Eppinga H, Peppelenbosch MP. Fumarates and Cancer. Trends Mol Med 2017;23:3-5.

29. Jiang S, Yan W. Succinate in the cancer-immune cycle.

Cite this article as: Huang Y, Yang X, Sun F, Lu T, Bi G, Liang J, Sui Q, Zhan C, Shi Y, Wang Q. Prognostic effects of glycometabolism changes in lung adenocarcinoma: a prospective observational study. Transl Lung Cancer Res 2019;8(6):808819. doi: $10.21037 /$ tlcr.2019.10.18
Cancer Lett 2017;390:45-7.

30. Ren JG, Seth P, Clish CB, et al. Knockdown of malic enzyme 2 suppresses lung tumor growth, induces differentiation and impacts PI3K/AKT signaling. Sci Rep 2014;4:5414. 


\section{Supplementary}

Table S1 Information sheet for standards of glucose metabolites

\begin{tabular}{|c|c|c|c|c|}
\hline Glucose metabolites & Identification of product & Company & Country & City \\
\hline Glucose-6-phosphate (G6P) & sc-221488 & Santa Cruz Biotechnology & United States & California \\
\hline Fructose-6-phosphate (F6P) & sc-202563 & Santa Cruz Biotechnology & United States & California \\
\hline Glucose-1-phosphate (G1P) & sc-296687 & Santa Cruz Biotechnology & United States & California \\
\hline Glyceraldehyde-3-phosphate (G3P) & sc-280680 & Santa Cruz Biotechnology & United States & California \\
\hline Dihydroxyacetone phosphate (DHAP) & sc-214896 & Santa Cruz Biotechnology & United States & California \\
\hline 3-phosphoglycerate (G-3P) & sc-214793 & Santa Cruz Biotechnology & United States & California \\
\hline 2-phosphoglycerate (2PGA) & sc-218005 & Santa Cruz Biotechnology & United States & California \\
\hline Lactate & 71718 & Sigma-Aldrich & United States & Louis \\
\hline Citrate & PHR1416 & Supelco & United States & Bellefonte \\
\hline Cis-aconitic acid & PHL83833 & Sigma-Aldrich & United States & Louis \\
\hline$\alpha$-ketoglutarate & 4102 & Millipore & United States & Massachusetts \\
\hline Succinate & W327700 & Sigma-Aldrich & United States & Louis \\
\hline Fumarate & D95654 & Sigma-Aldrich & United States & Louis \\
\hline Malate & 46940-U & Supelco & United States & Bellefonte \\
\hline Oxaloacetate & 07753 & Sigma-Aldrich & United States & Louis \\
\hline
\end{tabular}
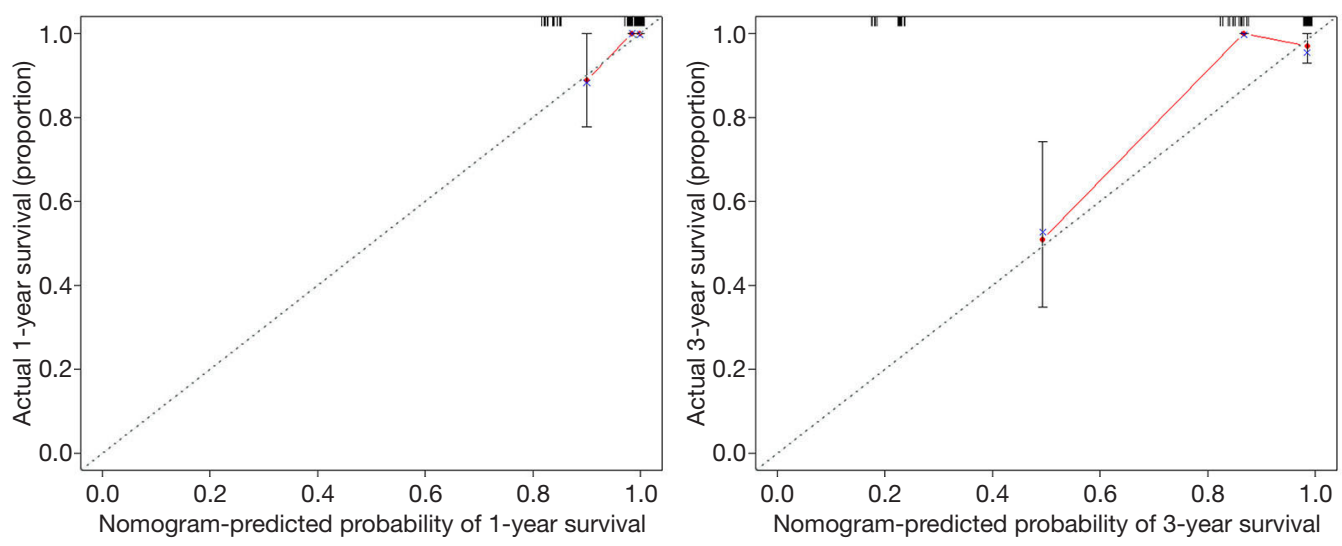

Figure S1 Calibration curves of the nomogram predicting 1- and 3-year overall survival of patients with lung adenocarcinoma. 
Table S2 Patient characteristics and results of univariate analyses of overall survival in the cohort

\begin{tabular}{|c|c|c|c|c|}
\hline \multirow{2}{*}{ Characteristics } & \multirow{2}{*}{$\mathrm{N}$} & \multicolumn{3}{|c|}{ Univariate } \\
\hline & & $\mathrm{HR}$ & $95 \% \mathrm{Cl}$ & $\mathrm{P}$ value \\
\hline Total & 100 & & & \\
\hline Age & & & & 0.717 \\
\hline$<65$ years & 63 & & & \\
\hline$\geq 65$ years & 37 & 1.21 & $0.43-3.40$ & \\
\hline Gender & & & & 0.649 \\
\hline Male & 45 & & & \\
\hline Female & 55 & 1.27 & $0.45-3.57$ & \\
\hline Tumor location & & & & 0.193 \\
\hline Left upper & 19 & & & \\
\hline Left lower & 28 & $19,555.8$ & $0-1.8 \mathrm{E}+85$ & 1.000 \\
\hline Right upper & 33 & $39,865.2$ & $0-3.7 E+85$ & 0.943 \\
\hline Right middle & 11 & $109,971.5$ & $0-1.0 \mathrm{E}+86$ & 0.939 \\
\hline Right lower & 9 & 1.0 & $0-6.6 E+142$ & 0.933 \\
\hline Smoking history & & & & 0.931 \\
\hline Never & 74 & & & \\
\hline Former/current & 26 & 1.05 & $0.34-3.30$ & \\
\hline T stage $(P)$ & & & & 0.057 \\
\hline $\mathrm{T} 1$ & 63 & & & \\
\hline $\mathrm{T} 2$ & 35 & 2.55 & $0.88-7.34$ & 0.084 \\
\hline T3 & 2 & 9.46 & $1.14-78.76$ & 0.038 \\
\hline$N$ stage $(P)$ & & & & $<0.001$ \\
\hline NO & 73 & & & \\
\hline N1 & 11 & 25.41 & $5.10-126.58$ & $<0.001$ \\
\hline N2 & 16 & 21.74 & $4.51-104.86$ & $<0.001$ \\
\hline M stage $(P)$ & & & & $<0.001$ \\
\hline MO & 97 & & & \\
\hline M1 & 3 & 19.40 & $5.17-72.80$ & \\
\hline Glucose & & & & 0.012 \\
\hline Lower & 48 & & & \\
\hline Higher & 52 & 6.77 & $1.53-30.01$ & \\
\hline Pyruvate & & & & $<0.001$ \\
\hline Lower & 78 & & & \\
\hline Higher & 22 & 20.40 & $5.72-72.77$ & \\
\hline Lactate & & & & 0.070 \\
\hline Lower & 36 & & & \\
\hline Higher & 64 & 3.96 & $0.89-17.56$ & \\
\hline Citrate & & & & 0.041 \\
\hline Lower & 40 & & & \\
\hline Higher & 60 & 4.71 & $1.06-20.88$ & \\
\hline Ketoglutarate & & & & 0.091 \\
\hline Lower & 39 & & & \\
\hline Higher & 61 & 0.41 & $0.15-1.15$ & \\
\hline Fumarate & & & & 0.549 \\
\hline Lower & 33 & & & \\
\hline Higher & 67 & 0.73 & $0.26-2.05$ & \\
\hline
\end{tabular}

